\title{
Management of progressive renal failure: the role of dietary manipulations
}

\author{
A.M. El Nahas \\ Northern General Hospital, Herries Road, Sheffield S5 7AU, UK.
}

In the majority of patients with chronic renal failure (CRF), the underlying nephropathy continues to progress well after the initiating events have subsided. ${ }^{\prime}$ As a result, renal function in these patients declines relentlessly with time as functional renal tissue is progressively destroyed. Such progressive decline in renal function occurs in most patients at a constant and predictable rate. ${ }^{2}$ This should allow for the testing of the efficacy of therapeutic interventions aimed at slowing the progression of CRF. Unfortunately, the development of such therapies has been hampered in the past by our limited knowledge of the pathophysiology of the underlying renal and glomerular scarring processes. Recently, major advances have been achieved in this field which might suggest more rational and successful therapies.

The progression of CRF is known to be affected by factors such as patients' age and sex, ${ }^{3}$ underlying disease, ${ }^{4}$ genetic profile and immune environment. ${ }^{5}$ Similarly, in individual patients the severity of proteinuria and systemic hypertension ${ }^{4}$ can influence the rate of progression of their nephropathy. So far the management of CRF has been confined to the symptomatic relief of uraemic symptoms, treatment of renal osteodystrophy and control of hypertension.

Recent observations by Brenner and his colleagues in experimental animals suggest that renal haemodynamic factors play a preponderant role in the initiation and progression of CRF; following an initial reduction in functional renal mass, compensatory haemodynamic changes take place in the remnant glomeruli. These are brought about by changes in glomerular arteriolar resistances. They are characterized by glomerular vasodilatation, hyperperfusion and hyperfiltration when the initial glomerular lesion is mild or absent $^{6}$ and by glomerular vasoconstriction with subsequent ischaemia when the vessels or glomeruli are severely damaged at the onset. ${ }^{7}$ Both an increase in glomerular blood flow or in glomerular vascular resistances could lead to a compensatory increase in

Correspondence: A.M. El Nahas, M.R.C.P., Ph.D.

Received: 5 March 1987 filtration rate secondary to intra-glomerular hydrostatic hypertension (Figure 1). Glomerular hypertension and hyperfiltration would damage the endothelium layer which in turn would stimulate platelet aggregation and glomerular capillary thrombosis. Such glomerular micro-atherosis, combined with subsequent ischaemic tubulo-interstitial atrophy and calcification, would lead to end stage renal failure.

The relevance of these experimental observations to CRF in man remains to be determined. However, they shed some light on the mechanisms of action of some dietary interventions aimed at slowing the progression of CRF. Such therapies could be directed at reversing the early compensatory glomerular haemodynamic adjustments and/or the prevention of the late glomerular cellular damage and subsequent glomerular thrombosis. Finally, interventions could also aim at preventing the destruction of the renal tubules and their calcification. Dietary manipulations designed to achieve some of these goals are currently being evaluated.

\section{Low protein diets}

As early as 1939, Farr \& Smadel showed that a low protein diet (LPD) can considerably reduce the severity of experimental CRF. ${ }^{8}$ Since then, their observations have been confirmed by many workers in a variety of models of CRF in the rat. ${ }^{9,10}$ In these animals, LPD reduces proteinuria, slows the progression of the underlying nephropathy and delays death from uraemia.' Such diet reverses the early compensatory glomerular hyperperfusion and hyperfiltration. ${ }^{11}$

In man, up to the late sixties, low protein diets were confined to the symptomatic management of terminal uraemic symptoms. More recently, Johnson et al. observed that such diet delays the progression of CRF and postpones the requirement for dialysis therapy. ${ }^{12}$ This was later confirmed by others who showed that diets low in protein and supplemented with essential amino acids $^{13}$ or their keto-analogues ${ }^{14}$ were equally 


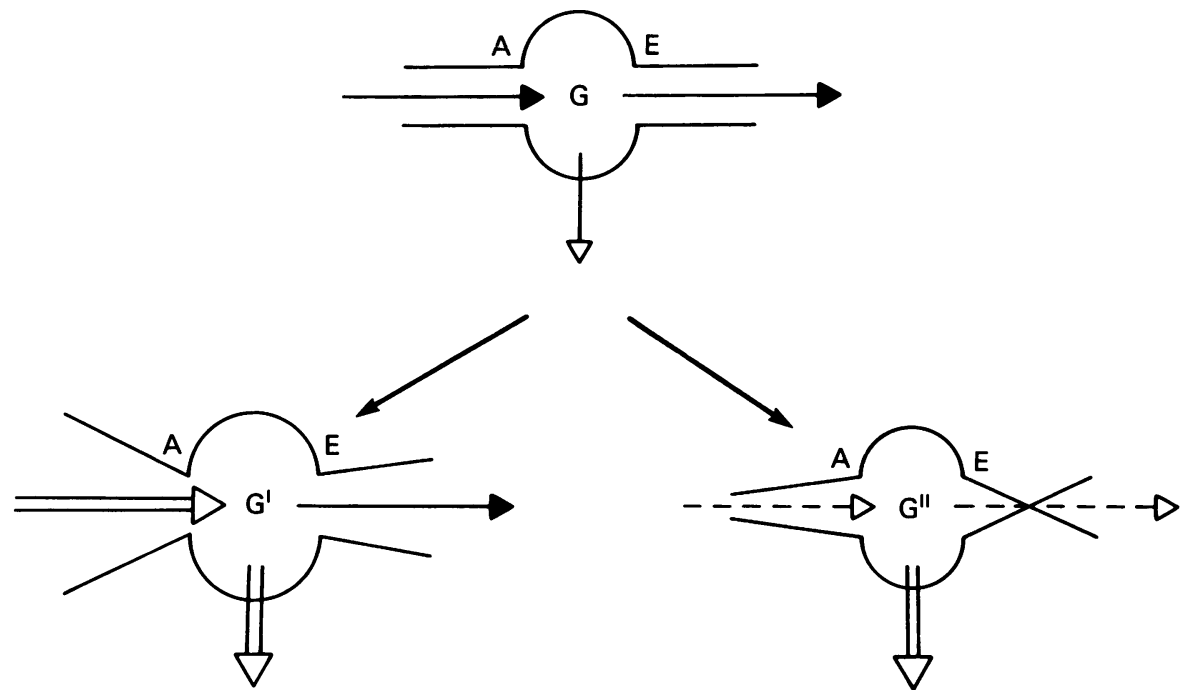

Figure 1 Compensatory glomerular haemodynamic alterations. G: normal glomerulus; G': hypertrophied, hyperperfused and hyperfiltrating glomerulus; $G^{\prime \prime}$ : atrophic, ischaemic and hyperfiltrating glomerulus; $A=$ afferent arteriolar resistance; $E=$ efferent arteriolar resistance. $G$ : In chronic tubulo-interstial nephritides; afferent $>$ efferent vasodilatation leading to compensatory intra-glomerular hvdrostatic hypertension with subsequent hyperfiltration ( $\forall)$. G": In chronic glomerulonephritides; etterent $>$ afferent vasoconstriction, leading to compensatory intra-glomerular hydrostatic hypertension with subsequent hyperfiltration $(\ddot{\nabla})$.

beneficial. It was also claimed that the early introduction of a LPD enhanced its efficacy; initiation of such dietary therapy was recommended when serum creatinine levels did not exceed $200 \mu \mathrm{mol} / \mathrm{l} .{ }^{15} \mathrm{We}$ noted that the beneficial effect of LPD was maximum in chronic tubulo-interstitial nephritides where the remnant glomeruli, as in their experimental counterparts, are relatively spared and capable of reversible hyperperfusion and hyperfiltration. Finally, we also observed that LPD reduces the magnitude of proteinuria in chronic glomerulonephritis. ${ }^{16}$ This was later confirmed by others in patients with nephrotic syndrome. ${ }^{17}$

While all these studies give reason for considerable hope and optimism, some doubts and controversy persist as to the potential benefit and side effects of LPD in the management of CRF. Briefly, a low protein intake decreases creatine and creatinine intake ${ }^{18}$ and might also interfere with their metabolism. ${ }^{19}$ Moreover, some degree of undernutrition ${ }^{20}$ with loss of muscle mass could also contribute iv a reduction in serum creatinine values. Unfortunately, the majority of studies conducted so far on the effects of LPD on progression of CRF have relied solely on serial estimations of serum creatinine as a marker of renal function. In view of the limitations of serum creatinine estimation on a low protein intake, these results and interpretations warrant some caution. ${ }^{21}$
Clearly, more studies are required where serial rens function assessments are made using isotopes. In the meanwhile, LPD $(0.5 \mathrm{~g} / \mathrm{kg}$ of body weight/day) should be restricted to the management of patients with advanced CRF (serum creatinine $>600 \mu \mathrm{mol}$ ). This should be done with careful monitoring of their nutritional status. ${ }^{21}$ Concomitantly, a high calorie intake should be encouraged $(>35 \mathrm{kcal} / \mathrm{kg} /$ day) in order to prevent catabolism and maximize the utilization of the limited protein supply. ${ }^{22}$

\section{Low phosphate diets}

In experimental animals, controversy has taken place over the actual benefit of low phosphate diets in CRF While some authors reported a considerable improvement in renal function on such diets ${ }^{23}$ others have attributed this effect to an overall reduction in food and protein consumption. ${ }^{24}$ However, more recent data have confirmed that a reduction in phosphate absorption by aluminium-containing resins decreases proteinuria, and protects rats from severe glomerulosclerosis. ${ }^{25}$ Similarly, the use of diphosphonates $^{26}$ as well as inhibitors of calcium phosphate precipitation and crystallization have proved beneficial in slowing progressive renal failure and preventing nephrocalcinosis. ${ }^{27}$ 
In man, most low protein diets are equally restricted in phosphate. It is therefore difficult to determine the respective benefit of nitrogen and phosphate on chronic renal failure. However, in one study, severe phosphate restriction $(<500 \mathrm{mg} / 24$ hours) was found to be synergistic with a low nitrogen diet in improving renal function..$^{28} \mathrm{~A}$ restricted phosphate intake (300 to $600 \mathrm{mg} / 24$ hours) should be supplemented by an adequate $(>1 \mathrm{~g} / 24$ hours) intake of calcium. In this respect calcium carbonate supplementation should prove suitable to increase calcium consumption and simultaneously decrease phosphate absorption. This would negate the need for aluminium containing phosphate binders with their inherent toxicity in CRF.

\section{Dietary lipids}

It was postulated by Moorhead et al. that the hyperlipidaemia of CRF and of patients with nephrotic syndrome, could accelerate the underlying renal scarring process. ${ }^{29}$ While confirmation of this hypothesis is awaited, many studies have examined the role of manipulations of dietary polyunsaturated fatty acids on the progression of experimental CRF; diets high in linoleic acid decrease experimental hypertension, reduce proteinuria and prevent severe renal histological damage. ${ }^{30}$ Changes in the dietary fish oil (eicosapentenoic acid - EPA) content of animals with CRF has so far led to conflicting results; while some reported an improvement, ${ }^{31}$ others showed an acceleration of the course of a model of experimental CRF ${ }^{32}$ Diets rich in polyunsaturated fatty acids are likely to have a dual effect on the glomerular scarring process; they could affect the early compensatory haemodynamic changes by modifying the levels of renal prostanoids, ${ }^{33}$ and they are likely to have an inhibiting effect on platelet aggregration ${ }^{34}$ and the late intra-glomerular thrombosis.

In man, diets supplemented with EPA have so far been shown to reduce the hyperlipidaemia and plasma viscosity of patients with CRF. ${ }^{35}$ These diets also reduce systemic blood pressure in essential hypertension. ${ }^{36}$ The role of such dietary manipulations on the progression of CRF in man is currently being evaluated.

\section{Dietary carbohydrates}

While most research has so far centred on the nephrotoxicity of dietary protein and phosphate. little attention has been paid to the effects of carbohydrates on the failing kidney. Limited available experimental, data suggest that a high sucrose intake accelerates the nephropathy of ageing rats, ${ }^{37}$ as well as the renal scarring in diabetic ${ }^{38}$ and sub-total nephrectomized ${ }^{39}$ animals. The relevance of these findings to the progression of CRF in man remains to be explored.

\section{Dietary monovalent and divalent cations}

In experimental models of CRF, restriction of dietary sodium intake has led to conflicting results; while some authors reported a reduction in proteinuria and a prevention of severe renal scarring in remnant kidneys and glomeruli upon dietary sodium restriction, ${ }^{40}$ others failed to confirm these data using a sodium free diet. ${ }^{41}$ In man, it is likely that dietary salt restriction, by influencing systemic blood pressure, might favourably affect the progression of CRF.

Little is known about the effects of dietary potassium manipulations on renal scarring. A high potassium intake, like a salt restricted one, could reduce systemic hypertension. ${ }^{42}$ However, in advanced CRF, the risks of hyperkalaemia might preclude dietary potassium supplementation.

Finally, the modification of calcium intake, like that of sodium and potassium, could influence systemic hypertension. ${ }^{42}$ In experimental CRF, a low calcium diet has proved as detrimental to the progression of the renal scarring as a high phosphate diet. ${ }^{43}$ In patients with CRF, calcium supplementation of their phosphate restricted diet is encouraged.

\section{Conclusions}

It is likely that, in the future, dietary manipulations in CRF will prove successful in slowing the progression of the underlying nephropathy. It is hoped that a better understanding of the pathophysiology of chronic renal scarring in man will lead to more rationalization of dietary therapy; dietary interventions might have to be tailored to the type and stage of the nephropathy treated. It is clear that more research and clinical trials are warranted before well defined and safe dietary guidelines could be drawn for patients with CRF.

\section{Acknowledgement}

I am grateful to Mrs D. Smith for her secretarial and editorial assistance. 


\section{References}

1. Kleinknecht, D., Grunfeld, J.P., Gomez, P.C., Moreau, J.F. \& Garcia-Torrez, R. Diagnostic procedures and long-term prognosis in bilateral renal cortical necrosis. Kidney Int 1973, 4: 390-396.

2. Mitch, W.E., Walser, M., Buffington, G.A. \& Lemann, J. A simple method of estimating progression of chronic renal failure. Lancet 1976, ii: 1326-1328.

3. Davison, A.M., Cameron, J.S., Kerr, D.N.S. et al. The natural history of renal function in untreated idiopathic membranous glomerulonephritis in adults. Clin Nephrol 1984, 22: 61-67.

4. Oldrizzi, L., Rugin, C., Valvo, E. et al. Progression of renal failure in patients with renal disease of diverse etiology on protein restricted diet. Kidnev Int 1985, 27: 553-557.

5. Cameron, J.S., Peters, D.K. \& Rees, A.J. New perspectives in glomerulonephritis. In Recent Advances in Renal Medicine 1982. 2: 1

6. Brenner, B.M., Meyer, T.W. \& Hostetter, T.H. Dietary protein intake and the progressive nature of kidney disease. N Engl J Med 1982, 307: 652-660.

7. Blantz, R.C., Tucker, B.J., Gushwa, L.C. et al. Glomerular immune injury in the rat: the influence of angiotensin II and $\alpha$-adrenergic inhibitors. Kidney Int 1981, 20: 452-461.

8. Farr, L.E., \& Smadel, J.E. The effects of dietary protein on the course of nephrotoxic nephritis in rats. J Exp Med 1939, 70: 615-627.

9. El Nahas, A.M., Paraskevakou, H., Zoob, S. et al. Effect of dietary protein restriction on the development of renal failure after sub-total nephrectomy in rats. Clin Science 1983, 65: 399-406.

10. Kleinknecht, C., Salusky, I., Broyer, M. et al. Effect of various protein diets in growth, renal function and survival in uraemic rats. Kidnev Int 1979, 15: 534-541.

11. Hostetter, T.H., Olson, J.L., Rennke, H.G., Venkatachalam, M.A. \& Brenner, B.M. Hyperfiltration in remnant nephrons: a potentially adverse response to renal ablation. Am J Physiol 1981, 241: F85-F93.

12. Johnson, W.J., Goldsmith, R.S., Towsey, P.P. et al. The influence of maintaining normal serum phosphate and calcium on renal osteodystrophy. In Norman, A.W. \& Schaefer, K. (eds) Vitamin D and Problems Related to Uraemic Bone Disease. Walter de Gruyten, New York, 1975, p. 573.

13. Bergström, J., Furst, P. \& Norée, L.O. Treatment of chronic uraemic patients with protein-poor diet and oral supply of essential amino acids. Clin Nephrol 1975, 3: 187-194.

14. Mitch, W.E., Walser, M., Steinman, T.E., Hill, S., Zeger, S. \& Tungasanga, K. The impact on the progression of chronic renal failure of a keto acid/amino acid supplement to a restricted diet. $N$ Engl J Med 1984, 311: 623-629.

15. Maschio, G., Oldrizzi, L., Tessitore, N. et al. Effects of dietary protein and phosphorus restriction in the progression of early renal failure. Kidney Int 1982, 22: $371-376$.

16. El Nahas, A.M., Master-Thomas, A., Brady, S.A. et al. Selective effect of low protein diets in chronic renal diseases. $\mathrm{Br}$ Med J 1984, 289: 1337-1340.

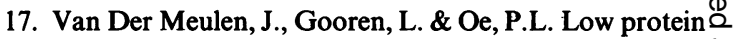
diet increases serum albumin by reducing proteinuria in $\subseteq$ some nephrotic patients. Proc Eur Dial Transplant Assoc $\overrightarrow{\bar{F}}$ Eur Ren Assoc 1985, 22: 1083-1086.

18. Bleiler, R.E. \& Schedl, H.P. Creatinine excretion; variability and relationships to diet and body size. $J \mathrm{Lab}$ 음 Clin Med 1962, 59: 945-955.

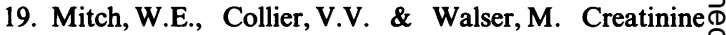
metabolism in chronic renal failure. Clin Sci 1980, 58: 327-335.

20. Lucas, P.A., Meadows, J.H., Roberts, D.E. \& Coles, $\vec{\circ}$ G.A. The risks and benefits of a low protein-essential amino acid-keto acid diet. Kidney Int 1986, 29: 995- $\vec{\omega}$ 1003.

21. El Nahas, A.M. \& Coles, G.A. Dietary treatment of chronic renal failure: Ten unanswered questions. Lancet $\underline{3}$ 1986, i: $597-600$.

22. Giordano, C. Protein restriction in chronic renal failure. Kidney Int 1982, 22: 401-408.

23. Ibels, L.S., Alfrey, A.C., Haut, L. \& Huffer, W.E. N Preservation of function in experimental renal disease by dietary restriction of phosphate. N Engl J Med 1978, 298: 122-126.

24. Laouari, D., Kleinknecht, C., Cournot-Witmer, G. et al. Beneficial effect of low phosphorous diets in uraemic rats: a reappraisal. Clin Sci 1982, 63: 539-548.

25. Lumlertgul, D., Alfrey, A., Burke, T.R. \& Schrier, R.ल Phosphate depletion independent of protein intake arrests progression of chronic renal failure. Kidney $\not t$ 1986, 29: 246(A).

26. Haut, L.L., Alfrey, A.C., Guggenheim, S. et al. Ren⿳亠口冋 toxicity of phosphate in rats. Kidney Int 1980, 17: 72 731.

27. Gimenez, L., Walker, G., Tew, W.P. \& Hermann, J.A. Prevention of phosphate induced progression of uraemia in rats by 3-phosphocitric acid. Kidney Int 1982, 22: 36-尺 41.

28. Barsotti, G., Giannoni, E., Morelli, E. et al. The decline 음 of renal function slowed by very low phosphorus intake in chronic renal failure patients following a low nitrogen diet. Clin Nephrol 1984, 21: 54-59.

29. Moorhead, J.F., El Nahas, A.M., Chan, M.K. \& Vargh-O ese, Z. Lipid nephrotoxicity in chronic progressive glomerular and tubulo-interstitial disease. Lancet 1982, ii: $1309-1311$.

30. Barcelli, U.O. \& Pollak, V.E. Is there a role for polyun- $\bigcirc$ saturated fatty acids in the prevention of renal disease 3 and renal failure. Nephron 1985, 41: 209-212.

31. Barcelli, U.O., Ito, Y., Miyata, J. et al. Influence of various dietary polyunsaturated fatty acids in rats with $\frac{D}{O}$ decreased renal mass. Clin Res. 1985, 33: 582A.

32. Scharschmidt, L., McGarry, L. \& Berger, P. The effects $N$ of fish oil eicosapentenoic acid as PGE synthesis in mesangial cells and in the $5 / 6$ nephrectomized rat. Kidney Int 1985, 27: 264A.

33. Bruckner, G.G., Lokesh, B., German, B. \& Kinsella, J.E. Biosynthesis of prostanoids, tissue fatty-acids composition and thrombotic parameters in rats fed diets enriched with docosahexenoic or eicosapentenoic acid. Thromb Res 1984, 34: 479-485

34. Sanders, T.A.B. Dietary fat and platelet function. Clin $\frac{T}{7}$ Sci 1983, 65: 343-350. 
35. Rylance, P.B., Gordge, M.P., Saynor, R. et al. Fish oil modifies lipids and reduces platelet aggregability in haemodialysis patients. Nephron 1986, 43: 196-202.

36. Norris, P.G., Jones, C.J.H. \& Weston, M.J. Effect of dietary supplementation with fish oil on systolic blood pressure in mild essential hypertension. $\mathrm{Br} \mathrm{Med} \mathrm{J} \mathrm{1986,}$ 293: $104-106$.

37. Cohen, A.M. \& Rosenmann, E. Diffuse intercapillary glomerulosclerosis in sucrose-fed rats. Diabetologia 1971, 7: 25-28.

38. Taylor, S.A., Cattell, V.C., Pacey, J. \& Price, R.G. Change in glomerular morphology in rats fed fructose diets. Similarity to diabetic microangiopathy. Proceedings International Symposium on Renal Basement Membranes, London 1985. (Abstracts)
39. Kleinknecht, C., Laouari, D., Hinglais, N. et al. Role of amount and nature of carbohydrates in the course of experimental renal failure. Kidney Int 1986, 30: 687-000.

40. Elema, J.D. \& Arends, A. Focal and segmental glomerular lyalinosis and sclerosis in the rat. Lab Invest 1975, 33: 554-561.

41. Purkerson, M.L., Hoffsten, P.H. \& Klahr, S. Pathogenesis of the glomerulopathy associated with renal infarction in the rat. Kidney Int 1976, 6: 407-417.

42. Kaplan, N.M. Non drug treatment of hypertension. Ann Int Med 1985, 102: 359-373.

43. Jobin, J.R. \& Bonjour, J.P. Compensatory renal growth: modulation by calcium PTH and $1,25-(\mathrm{OH})_{2} \mathrm{D}_{3}$. Kidney Int 1986, 29: 124-130. 